\title{
Membrane-seeded autologous chondrocytes: cell viability and characterization at surgery
}

\author{
Antonio Gigante - Claudia Bevilacqua • \\ Andrea Ricevuto • Monica Mattioli-Belmonte • \\ Francesco Greco
}

Received: 8 April 2005/ Accepted: 24 January 2006/ Published online: 13 June 2006

(C) Springer-Verlag 2006

\begin{abstract}
The implantation of chondrocytes, seeded on matrices such as hyaluronic acid or collagen membranes, is a method that is being widely used for the treatment of chondral defects. The aim of the present study was to evaluate the distribution, viability and phenotype expression of the cells seeded on a collagen membrane just at the time of the implantation. Twelve patients who were suffering from articular cartilage lesions were treated by the $\mathrm{MACI}^{\circledR}$ procedure. The residual part of each membrane was tested by colorimetric assay (MTT) and histochemical and ultrastructural analyses were carried out. In all of the samples a large number of viable cells, quite homogenously distributed, was detected. The cells expressed the markers of the differentiated hyaline chondrocytes. These data reassure in that the MACI procedure provides a suitable engineered tissue for cartilage repair, in line with the clinical evidences emerging in the literature.
\end{abstract}

Keywords Chondrocytes - Articular cartilage · Cells $\cdot$ Cultured $\cdot$ Histocytochemistry $\cdot$

Tissue engineering

\section{Introduction}

Autologous chondrocyte implantation (ACI) is an established method for the treatment of chondral defects [6]. Some prospective studies have demonstrated subjective and objective improvement in joint function at 12 months after surgery [3, 13]. MRI, second-look surgery and biopsies have shown the formation of nearly normal cartilage in a good number of patients $[13,18,19]$. On the basis of these results, ACI may be considered one of the most effective treatments of articular cartilage lesions. However, this method is not devoid of some problems with regard to, for example, the wide surgical approach, the demanding technique, the presence of periosteal flap and the cell-holding in site $[14,16]$.

In order to overcome some of these hurdles, new tissue engineering techniques, widely used nowadays, have been developed using chondrocytes seeded on biological matrices such as hyaluronic acid [15] or collagen membranes [7] or atelocollagen gel [17]. Despite the wide diffusion of these methods, there are still some areas that would need better clarification. The presence of numerous, viable and well-differentiated cells at surgery is an essential requisite for the success of all these methods. It has been shown that autologous chondrocytes in suspension before implantation are able to maintain their differentiated phenotype and are capable of proliferating fairly well $[9,20]$. On the other hand, little has been reported so far about cell characterization in membrane-seeded ACI techniques.

The aim of the present study was to evaluate the distribution, viability and phenotype expression of the cells seeded on a collagen membrane just at the moment of the implantation. 


\section{Materials and methods}

In 2003, 12 consecutive patients, 8 males and 4 females, mean age of 34 years, suffering from cartilage lesions of the knee (10 cases) and the ankle ( 2 cases), underwent a collagenic scaffold-based ACI procedure $\left(\mathrm{MACI}^{\circledR}{ }_{-}\right.$ Verigen, D). Autologous chondrocytes were isolated at the Verigen laboratories from cartilage slices obtained from non-bearing areas of the patients' joints during a preliminary arthroscopic surgery. Cells were propagated in monolayer cultures in autologous serum for 2 or 3 weeks, according to the cellular growth rate, and were passaged on average 3 times to obtain at least $10 \times 10^{6}$ cells. Cells were then seeded on $20 \mathrm{~cm}^{2}$ type $\mathrm{I} /$ III collagen membrane of porcine origin. The cell-seeded membranes were implanted in cartilage defects by means of either arthroscopic or mini-open surgery, using fibrin glue to ensure adhesion. At each implantation, the residual part of the membrane was collected and tested for cell viability, and histochemical and ultrastructural analyses were also performed.

\section{Cell viability analysis}

Cell viability was evaluated by MTT (dimethylthiazoldiphenyltetrazol bromide; thiazolil blue) colorimetric assay. MTT (Sigma, Italy) is a water soluble tetrazolium salt that yields a yellowish solution when prepared in medium lacking in phenol red (RTMI 1640, Sigma). Dissolved MTT is converted to an insoluble purple formazan by cleavage of the tetrazolium ring by the active mitochondrial dehydrogenases of living cells. The MTT solution (5 $\mathrm{mg}$ MTT/ml medium) was added to three samples $\left(1 \mathrm{~cm}^{2}\right)$ of each membrane, being assayed to equal $1 / 10$ of the original culture medium volume, and incubated for $3 \mathrm{~h}$. The solution was then removed and acidic isopropanol $(0.04-0.1 \mathrm{~N} \mathrm{HCl}$ in absolute isopropanol) was added to solubilize the stain.

The results were evaluated by means of the spectrophotometric assay $(570 \mathrm{~nm})$, yielding absorbance as a function of viable cell number.

\section{Histochemical and immunohistochemical analysis}

The samples were fixed by immersion in $4 \%$ paraformaldehyde in $0.1 \mathrm{M}$ phosphate buffer, $\mathrm{pH} 7.4$, at $4^{\circ} \mathrm{C}$ and then embedded in paraffin. Specimens were stained with safranin-O. For the immunohistochemistry, non-specific binding was blocked with $3 \%$ normal goat serum in a phosphate-buffered saline (PBS), $\mathrm{pH}$ 7.4 , for $30 \mathrm{~min}$ at room temperature; slides were then incubated overnight with primary antibodies at $4{ }^{\circ} \mathrm{C}$. Sections were incubated with polyclonal antibodies anti S-100 protein (Dako, Italy), a cytoplasmatic marker of chondrocyte phenotype, diluted at 1:3,000, anticollagen type I (Monosan, The Netherlands) and II (Calbiochem-Oncogene, CA, USA) at 1:150, and monoclonal antibodies anti chondroitin sulphate (chondroitin-S) (Sigma) at 1:200. Rabbit and mouse immunoglobulins, at the same dilutions as the primary antibodies, were used as controls. After three washes with Tris- $\mathrm{HCl}(0.05 \mathrm{M}, \mathrm{pH} 7.6)$, revelation of the reactions was accomplished by DAKO LSAB + kit, HRP. Stainings were viewed and photographed with a Leica Microscope (Leica Cambridge Ltd., UK).

\section{Ultrastructural analysis}

For scanning electron microscopy (SEM), the membranes were fixed in $2 \%$ glutaraldeyde in $0.1 \mathrm{M}$ cacodylate buffer ( $\mathrm{pH} 7.4$ ), post-fixed in $1 \%$ osmium tetroxide, dehydrated in increasing ethanol concentrations and then CPD-dried. They were mounted on stubs and gold-sputtered. Specimens were observed with a Philips 505 microscope.

\section{Results}

\section{Cell viability analysis}

The presence of numerous viable cells was observed in all samples, with quite a homogeneous stain distribution, even if some of the areas revealed a greater concentration of converted dye (Fig. 1).

Considerable variability was found in mean cell numbers between the samples from different patients, in relation to the different amounts of cells obtained from cartilage slices and seeded on the membrane.

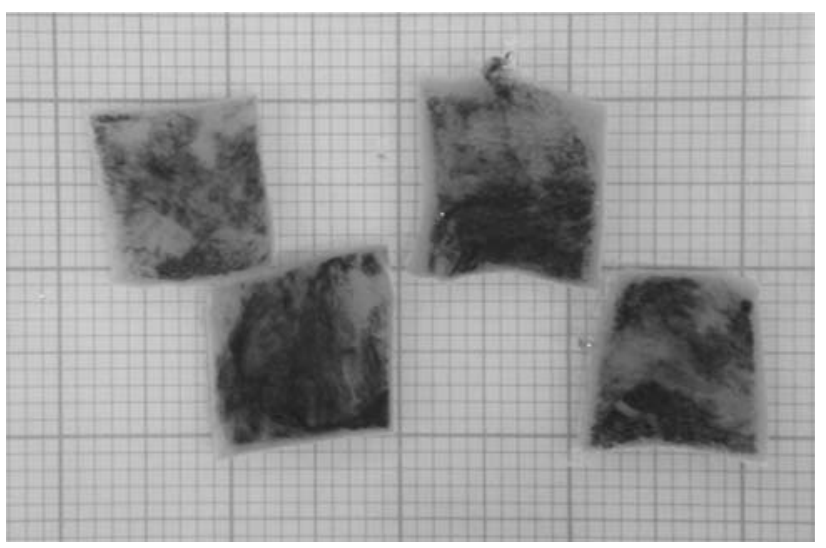

Fig. 1 Macroscopic view of human chondrocytes seeded on type I, III collagen membrane after MTT incubation. The converted blue dye shows the presence of numerous viable cells with quite a homogeneous stain distribution 
Highest values were observed in samples from the younger patients (Fig. 2, Table 1).

Histochemical and immunohistochemical analysis

In all of the samples, chondrocytes that were grown on the membrane were arranged in multi-layered sheets, and sometimes invaded deeper into the matrix. The cells appeared to be quite well differentiated, although some had a flattened morphology. They slightly stained metachromatically for safranin O (Fig. 3), and clearly immunoreacted with anti-S-100 protein (Fig. 4), type II collagen (Fig. 5) and chondroitin-S antibodies. Only a few cells stained for type I collagen.

\section{Ultrastructural analysis}

The membranes presented a dual appearance: there was a smooth side with tightly packed fibres and a rougher side, a sparse layer, to which the cells were adhered. Numerous cells firmly adhered to the membrane and showed a round shaped morphology and a rough surface (Fig. 6).

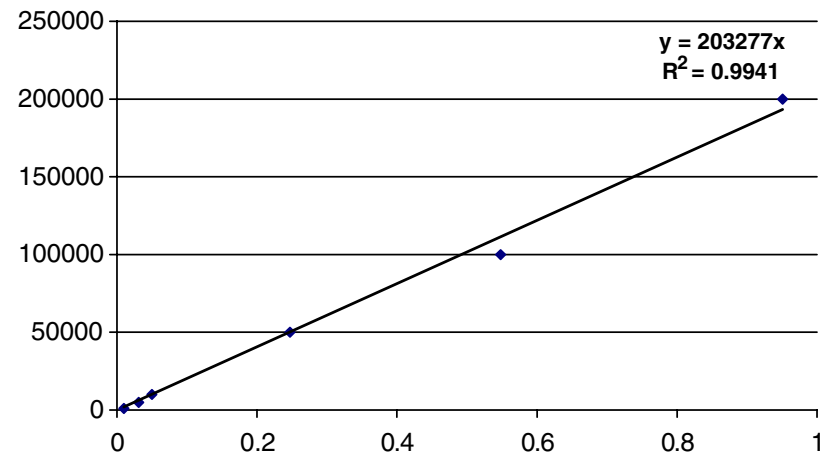

Fig. 2 The standard curve obtained from each membrane by MTT colorimetric assay (see also Table 1 )

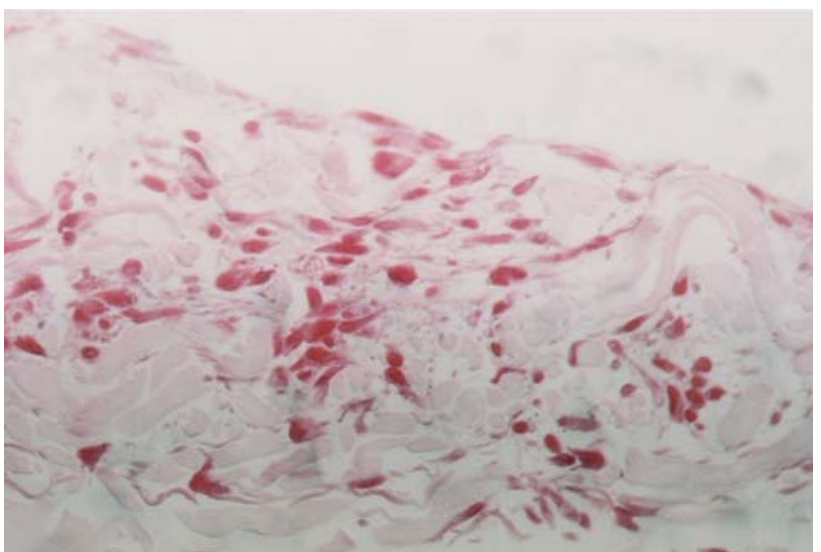

Fig. 3 Human chondrocytes seeded on type I, III collagen membrane before the implantation. Numerous cells are located on the membrane and deeper in the matrix forming a multi-layer engineered tissue. (Safranin O stain, $\times 200$ )

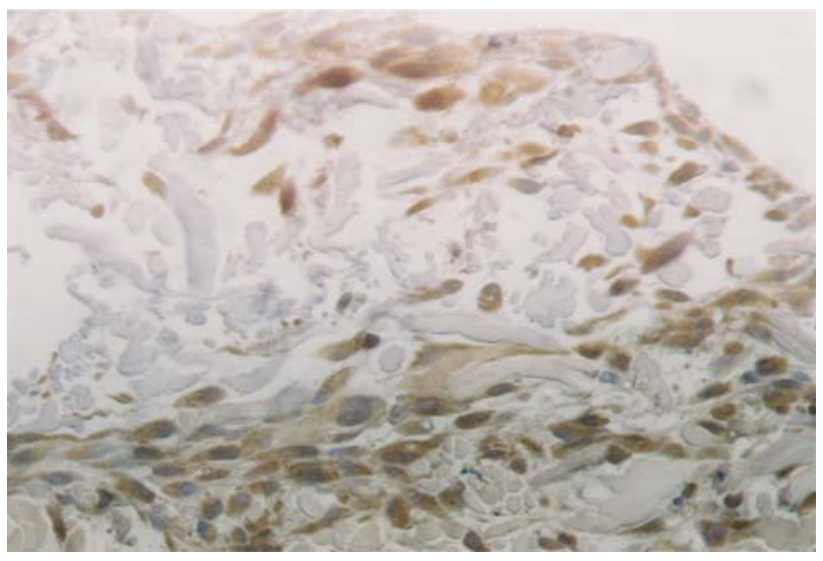

Fig. 4 Human chondrocytes seeded on type I, III collagen membrane. A marked cytoplasmic immunoreaction for S-100 protein is evident $(\times 400)$

\section{Discussion}

Cell phenotype and proliferation analysis should be an essential step in the evaluation of all tissue-engineered
Table 1 The table shows the mean viable cell number $/ \mathrm{cm}^{2}$ obtained from each membrane by MTT colorimetric assay

Patients' characteristics and the number of cells initially seeded on each membrane are also reported (see also Fig. 2)

\begin{tabular}{llllll}
\hline Case & Joint & Age & Sex & Seeded cells & Viable cells/cm ${ }^{2}(\mathrm{mean} \pm$ SD) \\
\hline 1 & Knee & 54 & M & $19 \times 10^{6}$ & $(7.52 \pm 2.79) \times 10^{3}$ \\
2 & Knee & 22 & F & $17 \times 10^{6}$ & $(12.94 \pm 2.54) \times 10^{3}$ \\
3 & Knee & 50 & F & $15.4 \times 10^{6}$ & $(17.82 \pm 6.25) \times 10^{3}$ \\
4 & Knee & 23 & M & $20.3 \times 10^{6}$ & $(100.28 \pm 22.14) \times 10^{3}$ \\
5 & Knee & 29 & F & $20.4 \times 10^{6}$ & $(110.07 \pm 39.18) \times 10^{3}$ \\
6 & Knee & 43 & M & $18.7 \times 10^{6}$ & $(5.34 \pm 3.25) \times 10^{3}$ \\
7 & Knee & 48 & M & $17.5 \times 10^{6}$ & $(4.74 \pm 2.37) \times 10^{3}$ \\
8 & Ankle & 42 & F & $18.2 \times 10^{6}$ & $(33.13 \pm 25.92) \times 10^{3}$ \\
9 & Knee & 19 & M & $21.6 \times 10^{6}$ & $(99.54 \pm 25) \times 10^{3}$ \\
10 & Knee & 35 & M & $20 \times 10^{6}$ & $(103.81 \pm 37.26) \times 10^{3}$ \\
11 & Ankle & 39 & M & $19 \times 10^{6}$ & $(79.48 \pm 62.57) \times 10^{3}$ \\
12 & Knee & 13 & M & $20.1 \times 10^{6}$ & $(87.88 \pm 55.17) \times 10^{3}$ \\
\hline
\end{tabular}




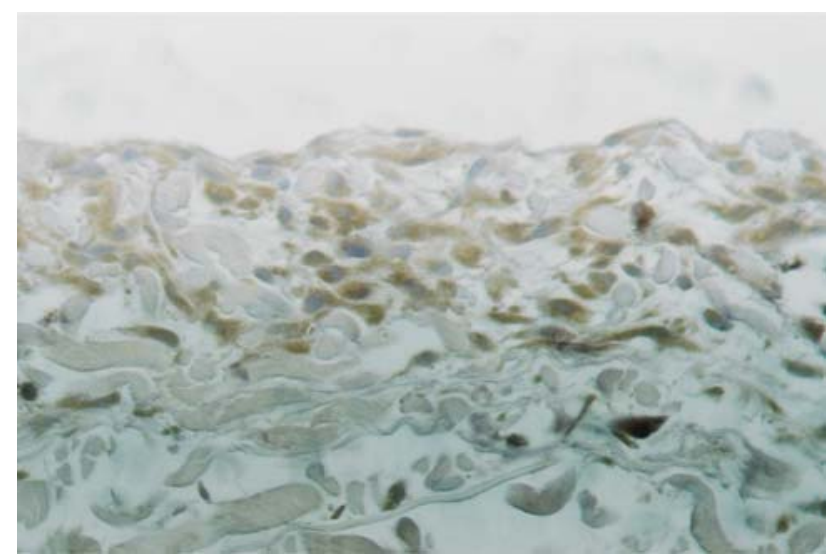

Fig. 5 Human chondrocytes seeded on type I, III collagen membrane. Cells express a positive immunoreaction for type II collagen, while the membrane remains unstained $(\times 400)$

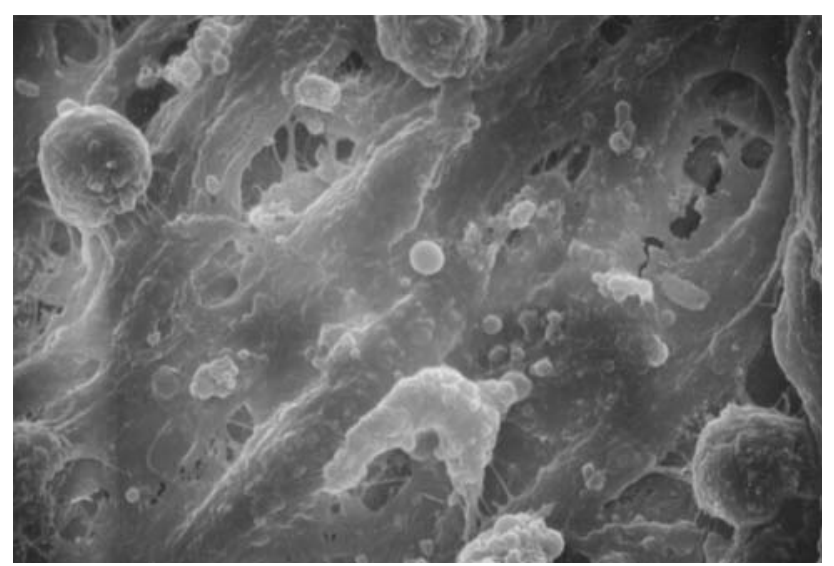

Fig. 6 Scanning electron micrograph of human chondrocytes seeded on type I, III collagen membrane. Numerous cells (arrows) firmly adhere to the membrane and show a round shaped morphology and a rough surface $(\times 7,500)$

products because the implantation of dedifferentiated or not-proliferating cells would not justify the therapeutic employment of these biotechnologies.

The chondrocyte culture may undergo a dedifferentiation process, consisting in a fibroblast-like morphology, a reduction of the type II collagen and aggregating proteoglycans synthesis, and an increase of type I collagen expression [1,4]. Chondrocyte dedifferentiation is common in monolayer cultures, while a long phenotype maintenance is supported by the presence of a tri-dimensional matrix $[1,4,11]$. The chemical and ultrastructural characteristics of the matrix may have great influence on the behaviour of chondrocytes in culture. A Type I collagen membrane appears to be a matrix endowed with such properties which make that ideal and useful for cartilage tissue engineering $[8,10]$.
MACI is a method for the treatment of articular cartilage defects, that employs autologous chondrocytes expanded in monolayer culture, suspended, and then seeded on type I-III collagen membrane. In a previous study [9], we had observed that autologous chondrocytes in suspension before implantation were of "good cartilaginous quality" and that they possessed an excellent proliferation capacity. Zheng et al. [20] confirmed that cells in ACI procedure maintained chondrocyte phenotype and showed a low apoptotic rate. To our knowledge, there is no previous reference to the analysis of the phenotype and the proliferation activity of human chondrocytes before MACI implantation. Data about cell number and viability, but not about cell phenotype and distribution are provided by Verigen's laboratory for each implant. It should, however, be remembered that it is quite possible that the presence of a membrane like the one found in the MACI technique could interfere with cell behaviour and modify phenotype expression.

The present study has demonstrated that the autologous chondrocytes that were analysed adhered to the membrane and had quite a homogenous distribution. These cells were viable in all the cases analysed, as shown by the spectrophotometric assay. The differences observed among patients in the number of cells seeded on the membrane and those found to be viable are probably due to several factors. In the first place, the amount of tissue collected from patients and sent to the laboratory for culture was variable. The cellularity of cartilage tissue and the proliferation capacity of chondrocytes in identical culture conditions are also quite variable between subjects, and this variability cannot merely be explained with age, but is more likely to stem from a number of characteristics that define tissue "quality" $[5,12]$.

We did not investigate whether during monolayer culture chondrocytes underwent dedifferentiation, however, other authors [20] demonstrated that cells grown in monolayer with the same culture conditions maintained a differentiated phenotype. Moreover, we felt it was important to establish the phenotypical expression of chondrocytes seeded on the I/III collagen membrane, since those are the cells that are then implanted, independently of what has taken place in the previous steps of the procedure. The positive immunostaining for S-100 protein, chondroitin-S, type II, but not type I collagen, confirms that cells maintained the characteristics of differentiated hyaline chondrocytes at the moment they were implanted.

These data reassure that the MACI procedure can indeed provide a suitable engineered tissue for cartilage repair, in line with the mid-term clinical results 
described in the literature so far [2, 7]. Nevertheless, clinical and histological results of this method to attain stable clinical recovery and a hyaline-like cartilaginous scar are auspicated to substantiate these evidences in the long term.

Acknowledgments The authors wish to thank Miss Sandra Manzotti of the Laboratory of the Department of Orthopaedics, Polytechnic University of Marche, Italy, for the preparation of the histological sections.

\section{References}

1. Archer CW, McDowell J, Bayliss MT, Stephens MD, Bentley G (1990) Phenotypic modulation in sub-populations of human articular chondrocytes in vitro. J Cell Sci 97:361-371

2. Bachmann G, Basad E, Lommel D, Steinmeyer J (2004) MRI in the follow-up of matrix-supported autologous chondrocyte transplantation (MACI) and microfracture. Radiologe 44:773-782

3. Bentley G, Biant LC, Carrington WJ, Akmal M, Goldberg A, Williams AM, Skinner JA, Pringle J (2003) A prospective, randomised comparison of autologous chondrocyte implantation versus mosaicplasty for osteochondral defects in the knee. J Bone Joint Surg [Br] 85-B:223-230

4. Benya PD, Shaffer JD (1982) Dedifferentiated chondrocytes reexpress the differentiated collagen phenotype when cultured in agarose gels. Cell 30:215-224

5. Bobacz K, Erlacher L, Smolen J, Soleiman A, Graninger WB (2004) Chondrocyte number and proteoglycan synthesis in the aging and osteoarthritic human articular cartilage. Ann Rheum Dis 63(12):1618-1622

6. Brittberg M, Lindahl A, Nilsson A, Ohlsson C, Isaksson O, Peterson L (1994) Treatment of deep cartilage defects in the knee with autologous chondrocyte transplantation. N Engl J Med 331:889-895

7. Cherubino P, Grassi FA, Bulgheroni P, Ronga M (2003) Autologous chondrocyte implantation using a bilayer collagen membrane: a preliminary report. J Orthop Surg (Hong Kong) 11:10-15

8. Ehlers E-M, Fub M, Rohwedel J, Russlies M, Kühnel W, Behrens P (1999) Development of a biocomposite to fill out articular cartilage lesions. Light, scanning and transmission electron microscopy of sheep chondrocytes cultured on a collagen I/III sponge. Ann Anat 181:513-518
9. Gigante A, Bevilacqua C, Zara C, Travasi M, Chillemi C (2001) Autologous chondrocyte implantation: cells phenotype and proliferation analysis. Knee Surg Sports Traumatol Arthrosc 9:254-258

10. Gigante A, Bevilacqua C, Cappella M, Manzotti S, Greco F (2003) Engineered articular cartilage: influence of the scaffold on cell phenotype and proliferation. J Mater Sci 14:713716

11. de Haart M, Marijnissen WJCM, van Osch GJVM, Verhaar JAN (1999) Optimization of chondrocyte expansion in culture. Acta Orthop Scand 70:55-61

12. Harrison PE, Ashton IK, Johnson WE, Turner SL, Richardson JB, Ashton BA (2000) The in vitro growth of human chondrocytes. Cell Tissue Bank 1(4):255-260

13. Henderson IJ, Tuy B, Connel D, Oakes B, Hettwer WH (2003) Prospective clinical study of autologous chondrocyte implantation and correlation with MRI at three and 12 months. J Bone Joint Surg [Br] 85-B:1060-1066

14. Jackson DW, Scheer MJ, Simon TM. (2001) Cartilage substitutes: overview of basic science and treatment options. J Am Acad Orthop Surg 9:37-52

15. Marcacci M, Zaffagnini S, Kon E, Visani A, Iacono F, Loreti I (2002) Arthroscopic autologous chondrocyte transplantation: technical note. Knee Surg Sports Traumatol Arthrosc 10:154-159

16. Messner K, Gillquist J (1996) Cartilage repair: a critical review. Acta Orthop Scand 67:523-529

17. Ochi M, Uchio Y, Kawasaki S, Iwasa J (2002) Trasplantation of cartilage-like tissue made by tissue engineering in the treatment of cartilage defects of the knee. J Bone Joint Surg Br 84(4):571-578

18. Peterson L, Minas T, Brittberg M, Nilsson A, Sjogren-Jansson E, Lindahl A (2000) Two- to 9-year outcome after autologous chondrocytes transplantation of the knee. Clin Orthop 374:212-234

19. Richardson JB, Caterson B, Evans EH, Ashton BA, Roberts S (1999) Repair of human articular cartilage after implantation of autologous chondrocytes. J Bone Joint Surg [Br] 81B:1064-1068

20. Zheng MH, King E, Kirilak Y, Huang L, Papadimitriou JM, Wood DJ, Xu J (2004) Molecular characterization of chondrocytes in autologous chondrocyte implantation. Int $\mathrm{J}$ Mol Med 13:623-628 